\title{
Effects of Electroacupuncture at Auricular Concha Region on the Depressive Status of Unpredictable Chronic Mild Stress Rat Models
}

\author{
Ru-Peng Liu, ${ }^{1}$ Ji-Liang Fang, ${ }^{1,2}$ Pei-Jing Rong, ${ }^{1}$ Yufeng Zhao, ${ }^{3}$ Hong Meng, ${ }^{1}$ Hui Ben, \\ Liang Li, ${ }^{1}$ Zhan-Xia Huang, ${ }^{4}$ Xia Li, ${ }^{4}$ Ying-Ge Ma, ${ }^{3}$ and Bing Zhu ${ }^{1}$ \\ ${ }^{1}$ Institute of Acupuncture and Moxibustion, China Academy of Chinese Medical Sciences, Beijing 100700, China \\ ${ }^{2}$ Guanganmen Hospital, China Academy of Chinese Medical Sciences, Beijing 100053, China \\ ${ }^{3}$ Clinical Evaluation Center, Institute of Basic Research in Clinical Medicine, China Academy of Chinese Medical Sciences, \\ Beijing 100700, China \\ ${ }^{4}$ Beijing University of Chinese Medicine, Beijing 100029, China
}

Correspondence should be addressed to Pei-Jing Rong; rongpj@mail.cintcm.ac.cn

Received 24 August 2012; Revised 21 November 2012; Accepted 14 December 2012

Academic Editor: Wolfgang Schwarz

Copyright (C) $2013 \mathrm{Ru}$-Peng Liu et al. This is an open access article distributed under the Creative Commons Attribution License, which permits unrestricted use, distribution, and reproduction in any medium, provided the original work is properly cited.

\begin{abstract}
To explore new noninvasive treatment options for depression, this study investigated the effects of electroacupuncture (EA) at the auricular concha region (ACR) of depression rat models. Depression in rats was induced by unpredictable chronic mild stress (UCMS) combined with isolation for 21 days. Eighty male Wistar rats were randomly assigned into four groups: normal, UCMS alone, UCMS with EA-ACR treatment, and UCMS with EA-ear-tip treatment. Rats under inhaled anesthesia were treated once daily for 14 days. The results showed that blood pressure and heart rate were significantly reduced in the EA-ACR group than in the UCMS alone group or the EA-ear-tip group. The open-field test scores significantly decreased in the UCMS alone and EA-ear-tip groups but not in the EA-ACR group. Both EA treatments downregulated levels of plasma cortisol and ACTH in UCMS rats back to normal levels. The present study suggested that EA-ACR can elicit similar cardioinhibitory effects as vagus nerve stimulation (VNS), and EA-ACR significantly antagonized UCMS-induced depressive status in UCMS rats. The antidepressant effect of EA-ACR is possibly mediated via the normalization of the hypothalamic-pituitary-adrenal (HPA) axis hyperactivity.
\end{abstract}

\section{Introduction}

Vagus nerve stimulation (VNS) was approved by the U. S. Food and Drug Administration in 2005 and has been frequently used as a treatment option for treatment-resistant depression (TRD) [1-4]. Its mechanisms of antidepressant action are not fully elucidated; however, its neuromechanisms are based on the direct stimulation of the cervical trunk of the left vagus nerve. The afferent fibers of vagus nerve project to solitary nucleus (SN). Fibers of SN project to the neuroendocrine systems in the limbic system structures and the autonomic nervous system. These areas are strongly interconnected by monoamine-related pathways, including the ventral tegmental area, brainstem, the hypothalamus, thalamus, amygdala, anterior insula, nucleus accumbens, and the lateral prefrontal cortex [5]. Furthermore, the ventral tegmental area has a dense dopaminergic input to the prefrontal cortex; fibers from the SN project to the locus ceruleus and dorsal raphe nucleus which are major brainstem nuclei related to noradrenergic (NE) and serotonergic (5HT) innervations of the entire brain cortex, respectively. It is well known that the serotonergic, dopaminergic, and noradrenergic systems are commonly involved in the pathophysiology of depression and in the neuromechanisms of action of antidepressants [6].

Nonetheless, typical implantation of VNS device requires an invasive surgical procedure which may be accompanied by some side effects, such as infection of wound, hoarse voice, dyspnea, difficulty swallowing, neck pain, paresthesia, emesis, laryngospasms, dyspepsia, cardiac asystole, bradycardia, 
and even heart failure. Worse still, technical complications of device malfunction may aggravate patient conditions $[7,8]$.

Enlightened by the mechanism of VNS, researchers in our team found that auricular concha region (ACR) is densely innervated by free nerve endings of the vagus nerve. Our previous animal studies found that electroacupuncture (EA) at ACR (EA-ACR) had significant effects in the management of primary hypertension $[9,10]$, diabetes mellitus $[11,12]$, and partial epilepsy $[13,14]$. EA-ACR is a noninvasive procedure which requires a portable EA device and no side effects. With a similar mechanism to VNS, EA vagus nerve stimulation may provide beneficial effects in the treatment of depression.

EA-ACR is one of the acupuncture therapeutic methods which can be considered as auriculotherapy. Theories of auriculotherapy dates back to 2000 years ago as first mentioned in the book of Yellow Emperor's Canon of Medicine (Huang Di Nei Jing) [15]. Modern auriculotherapy with 42 points was firstly introduced by Dr. P. Nogier (France) in 1956. The international standard map of auricularpoints was published in China and later was recommended by WHO in 1993. Acupuncture, part of the oriental medicine, has been used in eastern Asian countries for the management of various emotional, psychological, and psychiatric disorders including anxiety, stress, insomnia, and depression. In recent years, acupuncture has become one of the most popular complementary therapies in the West, and the therapeutic effectiveness of acupuncture on depression has been confirmed by modern research studies. Allen et al. [16] found that body acupuncture and auriculotherapy could significantly reduce the severity of depression. Similar results were demonstrated in the studies of Luo et al. [17] and Zhang et al. [18], in which researchers found that electroacupuncture was as efficacious as fluxetine in the management of major depression. In general, increasing evidences support that acupuncture is an effective treatment for patients with depressive disorders [1923].

In the current study, we aimed to verify the vagus nerve responses during EA at ACR. Furthermore, antidepression effects of EA-ACR were investigated by observation of behaviors and measurement of blood biochemicals in the rat models of unpredictable chronic mild stress (UCMS).

The results will provide a fundamental evidence for the anti-depression effects of EA-ACR and will facilitate EAACR to become a new noninvasive and low-cost therapy for depression.

\section{Methods and Materials}

2.1. Animals. Male Wistar rats in 150-170 g were obtained from the Laboratory Animal Resources Center, National Institute for the Control of Pharmaceutical and Biological Products, Beijing (Certificate no. SCXK (jing) 2009-0017). These animals were individually caged on a $12 \mathrm{~h}$ light/dark cycle (lights on at 8:00 a.m., lights off at 8:00 p.m.) under controlled temperature $\left(22 \pm 1^{\circ} \mathrm{C}\right)$ and humidity $(50 \% \pm$ $5 \%)$ conditions. Standard rat chow and water were given ad libitum. Animals were allowed to acclimatize for seven days before the study. All experiment procedures comply with the guidelines of the "Principles of Laboratory Animal Care" (NIH publication number 80-23, revised 1996) and the legislation of the People's Republic of China for the use and care of laboratory animals. The experimental protocols were approved by the Animal Experimentation Ethics Committee of the Institute of Acupuncture and Moxibustion, China Academy of Chinese Medical Sciences. Efforts were made to minimize the number of animal use and the suffering of the experimental animals.

2.2. Open Field Test for Behavioral Scoring. The open field apparatus was constructed of black plywood and measured $80 \times 80 \mathrm{~cm}$ with $40 \mathrm{~cm}$ walls. White lines were drawn on the floor. The lines divided the floor into twenty-five $16 \times 16 \mathrm{~cm}$ squares. A central square $(16 \mathrm{~cm} \times 16 \mathrm{~cm})$ was drawn in the middle of the open field. Rats were put on the central square, at the same time the video camera was turned on for video recording from the top of the open field apparatus. Behaviors of rats were recorded for 3 minutes, with the grid number being counted as the horizontal score and the time of both frontal claws uplifting from the ground as the vertical score. The total locomotor activity of each animal was then scored as the sum of the number of line crosses and rears [24, 25].

\subsection{Unpredictable Chronic Mild Stress (UCMS) Model. Eighty} Rats were evenly randomized into 4 groups. Forty-two rats were recruited with the total score of 30-120 in the open field test [25]. A successful UCMS model rat was created with the score of the open field test equal or minus 60. Qualified rats were distributed into four groups: the normal control $(n=10)$, UCMS alone $(n=8)$, UCMS with EA-ACR treatment (EA-ACR) $(n=12)$, and UCMS with EA-ear-tip as the treatment control (EA-ear-tip) $(n=12)$. Every five rats in the normal group were housed in one cage. However, rats in the UCMS alone, EA-ACR, and EA-ear-tip groups were caged individually. Depression model was established by 21 days of UCMS combined with isolation. UCMS procedures were based on published studies [25, 26], including seven kinds of stressors: food deprivation, water deprivation, cage tilt $45^{\circ}$ (Ugo Basile s.r.l. hot/cold plate, Model 35100-001, Italy), swimming in $4^{\circ} \mathrm{C}$ ice water, clipping tail $3 \mathrm{~min}, 50 \mathrm{~V}$ electric shock (Electronic stimulator, NIHON KOHDEN, Japan), and overnight illumination. The stressors were given randomly 3 times daily for 21 continuous days. The rats in the normal control group were housed undisturbedly except for necessary procedures such as routine cage cleaning.

2.4. Experimental Procedures (Figure 1). The open field test on all rats was conducted on the day before the study, the 22th day (after UCMS), the 36th day (after treatment), and the 50th day in the study course. After the models of UCMS were established in 21 days, the EA treatment of 14 days was applied to the bilateral auricular concha region (Figure 1) of rats in the EA-ACR group once daily for $20 \mathrm{~min}$. For the EA-ear-tip group, the EA applied to the bilateral ear tips (Figure 1) followed the same procedure and EA parameters as the EA-ACR. All rats in the EA groups 
accepted the inhaled anesthesia during the treatment. EA was set at the frequency of $2 \mathrm{~Hz}$, the intensity of $1 \mathrm{~mA}$ by using the electroacupuncture stimulator (HANS-100A, Nanjing Gensun Medical Technology Co., Ltd., China). The inhaled anesthesia was conducted on the ISOFLURANE VAPORIZER (Matrx VIP 3000, Midmark corporation, USA) with isoflurane (Hebei Nine Sent Pharmaceutical Co., Ltd., Heibei, China). Blood pressure, including systolic, diastolic and mean pressures, and heart rate of rats were monitored noninvasively by using the apparatus (BP-98A, Beijing Soft Long Biological Technology Co., Ltd., China) during one EA treatment/anesthesia. The data were recorded in numerical values at the starting point of anesthesia ( 0 min pre-EA), the 1st min of anesthesia (EA begins), the 6th min of anesthesia (EA $5 \mathrm{~min}$ ), the 11th min of anesthesia (EA $10 \mathrm{~min}$ ), the 16th min of anesthesia (EA $15 \mathrm{~min}$ ), and the 21st min of anesthesia (EA $20 \mathrm{~min}$ ) on the same day for three UCMS groups. At the 51st day of the study, the rats were sacrificed and their neck venous blood was sampled for the tests of plasma cortisol (ELISA, R\&D, USA) and ACTH (Acthlisa, R\&D, USA) levels.

2.5. Statistical Analysis. The statistical analysis was performed by using one-way analysis of variance (ANOVA) followed by a Turkey test with software SPSS 13.0. $P<0.05$ which was considered statistically significant, and the data were expressed as means \pm standard deviation.

\section{Results}

3.1. Effects of EA-ACR Treatment on Heart Rate and Blood Pressure in UCMS Rats (Figures 2 and 3). No statistical difference was found in heart rate and blood pressure among rats of the three UCMS groups at the beginning of the study. Both of the heart rate and blood pressure in three UCMS groups showed a descending trend during the anesthesia period. However, the two EA treatment groups reduced heart rate and blood pressure significantly compared to the UCMS alone group. The mean heart rate from the 6th to 11th min decreased significantly in the EA-ACR group compared to the EA-ear-tip group; furthermore, the mean blood pressure was downregulated significantly in the EA-ACR group compared to the EA-ear-tip group in the treatment period. The EA-ACR treatment resulted in a significant decrease in the heart rate between the starting point of anesthesia and the 11th min of anesthesia $(P<0.05)$ and in the mean pressure between the starting point of anesthesia and the 16th min of anesthesia $(P<0.05)$.

3.2. The Different Influences of EA-Treatments on the Open Field Test Score of UCMS Rats (Figure 4). The total score of rats exposed to the open field test showed a significant decrease on the 22th day compared to the beginning in both the UCMS alone group and the EA-ear-tip group $(P<0.01$ and $P<0.01$, resp.). However, the score did not show remarkable differences between the two time spots in the normal group or in the EA-ACR group, respectively. But the EA-ACR group reached the score of 60 in the open field test and was thus qualified as the UCMS model. In the EA-eartip group, the score decreased significantly on the 36th day compared to the 22 nd day.

\subsection{Effects of EA Treatment on Plasma Cortisol and ACTH} Levels in UCMS Rats (Figures 5 and 6). As compared with the normal group, plasma cortisol levels in the three UCMS groups showed significant increases $(P<0.01$ for all comparisons). On the other hand, the mean plasma cortisol level of the EA-ACR group and the EA-ear-tip group decreased significantly compared to the UCMS alone group $(P<0.05$ and $P<0.05$, resp.).

The 21-day UCMS exposure significantly increased the concentration of ACTH in rat blood $(P<0.01)$ when the UCMS group was compared with the normal control on the 51st day of the study. However, EA treatments significantly decreased the concentration of ACTH compared to the UCMS alone group $(P<0.01$ and $P<0.01$, resp. $)$, while none of the EA treatment groups showed a significant difference in the concentration of ACTH as the normal control group did.

\section{Discussion}

In general, EA treatments down-regulated the heart rate and blood pressure as well as the concentration of plasma cortisol and ACTH. However, the heart rate and blood pressure were influenced more intensively by the EA-ACR than the EA-eartip, and the open field test score was kept at a higher level by EA-ACR only.

4.1. Cardioinhibitory Effects of EA-ACR Are Similar to the Vagus Nerve Stimulation. In the present study, EA-ACR elicited a significant decrease in heart rate and mean pressure under the anesthesia; however, the EA-ear-tip treatment did not induce similar changes during the treatment. These results suggested that EA stimulation at the auricular concha region induced similar effects as that of the direct vagus nerve electric stimulation had on the heart $[7,27,28]$. VNS, which stimulates the cervical trunk of the vagus nerve directly, is a procedure that was approved by FDA to treat primary hypertension years ago [7,27]. Our previous research showed that acupuncture at auricular concha area could effectively decrease essential hypertension in rat models [9]. In addition, we found that electric stimulation on nucleus dorsalis nerve vagi could induce immediate decrease in heart rate and corresponding changes of electrocardiogram [29].

Anatomical knowledge of the vagus nerve informs that the auricular fibers of vagus nerve densely distribute in the concha and external auditory meatus of the ear; however, there are a few vagus nerve fibers around the ear tip [30]. The auricular branch of the vagus nerve ascends to the superior vagal ganglion (nucleus dorsalis nerve vagi), where the cholinergic preganglionic parasympathetic neurons give rise to the branchial efferent motor fibers innervating the heart, and stimulating pathway induces cardioinhibitory effects [28]. Afferent signals elicited by EA-ACR may be integrated at the medulla oblongata, which then generates regulatory signals to activate the cardiac vagus nerve. Cardiac vagus 


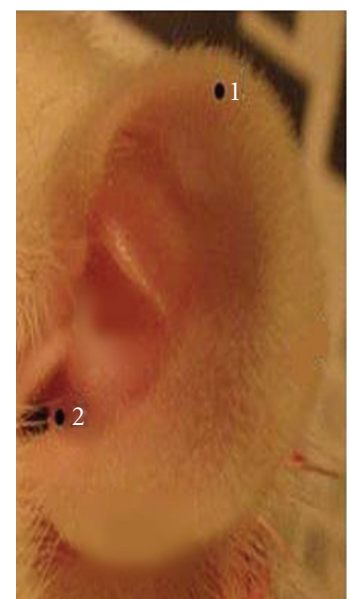

FIGURE 1: Electrical stimulation spot. Black spot 1 on the ear-tip area (nonauricular concha control). Black spot 2 on the auricular concha region (ACR). Positive iron pole (diameter $0.3 \mathrm{~mm}$ ) on the frontal side of ear, and the negative iron pole (diameter $0.3 \mathrm{~mm}$ ) on the back side.

nerve activation slows the heart rate and decreases the blood pressure immediately.

\subsection{EA-ACR Treatment Improved the Depressive Status of} UCMS Rats in the Open Field Test. The unpredictable chronic mild stress (UCMS) has already contributed to the elucidation of the pathophysiological mechanisms of depression such as decreased neurogenesis and HPA axis alterations $[26,31]$. In the current study, this model was used to explore the relations between depressive-like behavior in rats and EAACR treatment. The open field test provides simultaneous measurement of UCMS. A higher score in the test indicates increased locomotion and exploration and/or a lower level of anxiety [25, 26, 31]. In our study, the scores of open field test were significantly decreased in the UCMS alone and EA-ear-tip groups on day 22 compared to the beginning date, but no significant decrease was found in the EAACR group. However, all the rats in the EA-ACR group were qualified for modeling with a standard recruiting score of 60 . It was apparent that EA-ACR kept the score on a higher level in the treatment course, while the score of the EA-ear-tip group showed a significant decrease during the treatment. This phenomenon indicated that EA-ACR induced the antidepressive effects. The UCMS model and the open field test were also successfully introduced into previous EA studies on depression. For example, EA at Baihui (GV20) and Yingtang (EX-HN3) on the top and front head scalp for 21 days can significantly improve the symptom of the depressive rats, the crossing and rearing movement times, and the number of p-CREB-positive neuron in the hippoeampus as the fluoxetine compared with the UCMS alone group [25].

4.3. EA Treatment Normalized the Hyperactivity of Hypothalamic-Pituitary-Adrenal (HPA) Axis. In the present study, 21 days' UCMS exposure significantly increased the concentrations of plasma cortisol and ACTH in rats. It is

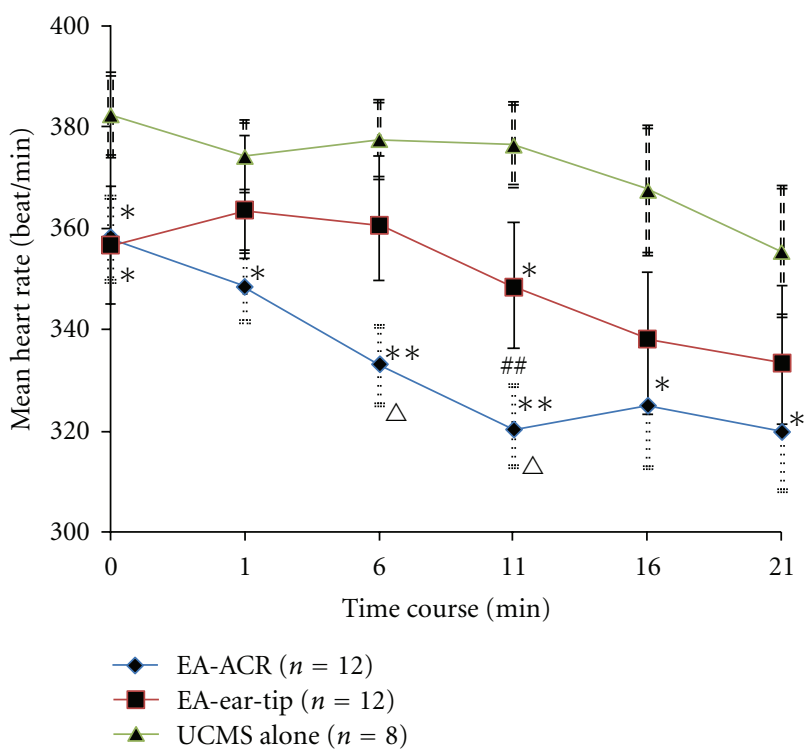

FIGURE 2: The time course of heart rate for three UCMS groups during one EA treatment/anesthesia. Comparison between the EAtreated UCMS group and the UCMS alone group, ${ }^{*} P<0.05$, ${ }^{* *} P<0.01$. Comparison between the EA-ACR group and the EAear-tip group, ${ }^{\triangle} P<0.05,{ }^{\triangle} P<0.01$. Comparison between the starting point of anesthesia and the 11th min in the EA-ACR group. ${ }^{\# \#} P<0.01$.

consistent with the previous research studies on both human beings and animal models with depressive status $[32,33]$. Furthermore, 14 days of EA treatments (both EAACR and EA-ear-tip) right after UCMS down-regulated the plasma cortisol and ACTH in UCMS rats to normal levels. Researchers found that EA at acupoints Neiguan(PC6), Sanyinjiao(SP6), and Taichong(LV3) can lower plasma cortisol and ACTH levels and improve symptoms in depression [33].

Several hypotheses have been proposed for the pathological mechanism of depression. Besides disturbed monoaminergic neurotransmission, hyperactivity of hypothalamicpituitary-adrenal (HPA) axis is closely related to major depression [34-36]. The HPA axis is the primary neuroendocrine system responsible for coordinating the mammalian stress response and has thus been a major focus of neurobiological research of depression. Major components of the HPA axis include corticotropin-releasing factor (CRF), adrenocorticotropin hormone (ACTH), and glucocorticoids. Cortisol is the major glucocorticoid in humans and animals. During stress response, neurons in the paraventricular nucleus (PVN) of the hypothalamus release CRF into the hypothalamo-pituitary portal system. CRF then stimulates the release of adrenocorticotropin (ACTH) from the anterior pituitary into systemic circulation, which in turn stimulates the adrenal cortex to secrete cortisol. Cortisol is responsible for many of the physiological changes associated with the stress response, and it also provides negative feedback to the hypothalamus and pituitary to decrease the synthesis and release of CRF and ACTH. 


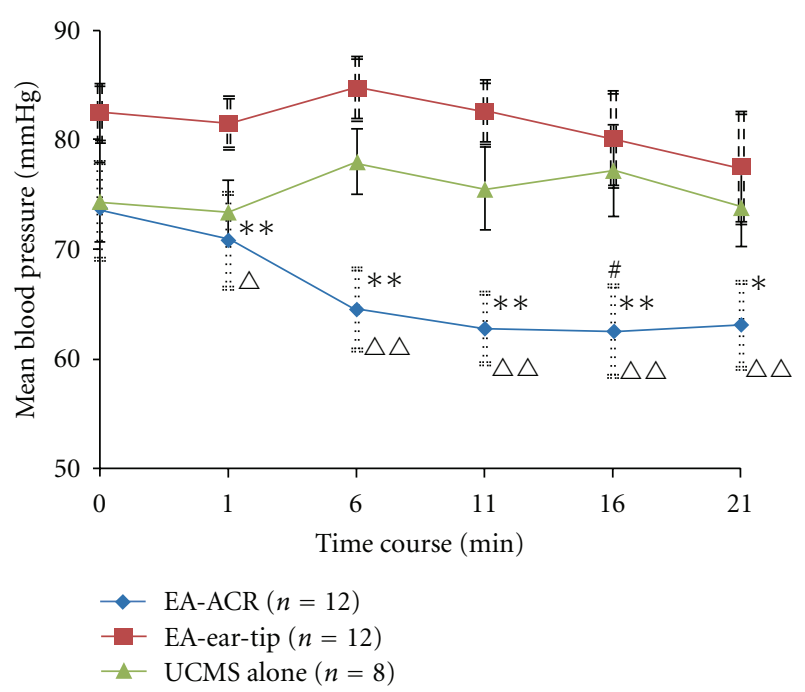

FIGURE 3: The time course of the mean blood pressure for the three UCMS groups during one EA treatment/anesthesia. Comparison between the EA-treated UCMS group and the UCMS alone group, ${ }^{*} P<0.05,{ }^{* *} P<0.01$. Comparison between the EA-ACR group and the EA-ear-tip group, ${ }^{\triangle} P<0.05,{ }^{\triangle} P<0.01$. Comparison between the starting point of anesthesia and the 16th min in the EAACR group. ${ }^{\#} P<0.05$.

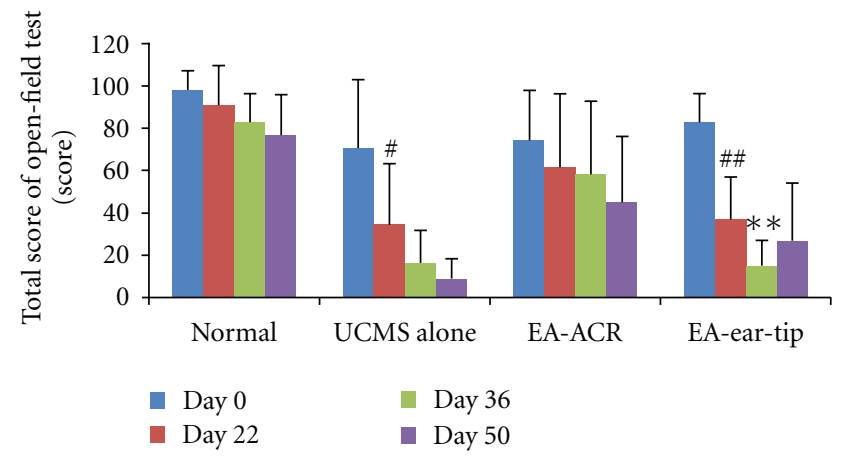

Figure 4: The influence of EA-ACR on the open field test score of rats. The 22 nd day compared with the day before the test (day 0 ) in the same group, ${ }^{\#} P<0.05,{ }^{\# \#} P<0.01$; the 36 th day compared with the 22nd day in the EA-ear-tip group, ${ }^{* *} P<0.01$.

Patients with depression show hyperactivity of the HPA axis that may result from the impaired negative feedback regulation of glucocorticoid release [34]. Moreover, research study also found that normalization of these HPA axis abnormalities is associated with successful antidepressant treatment, and patients whose HPA abnormalities do not normalize are significantly more likely to relapse [37]. In a VNS treatment study, O'Keane et al. [38] found that the $\mathrm{CRF} / \mathrm{ACTH}$ (adrenocorticotropic hormone) responses in the depressed group before VNS implantation were significantly higher than in the healthy group and were reduced to normal values after 3 months of VNS treatment; in addition, they also found significant improvement in depression symptoms.

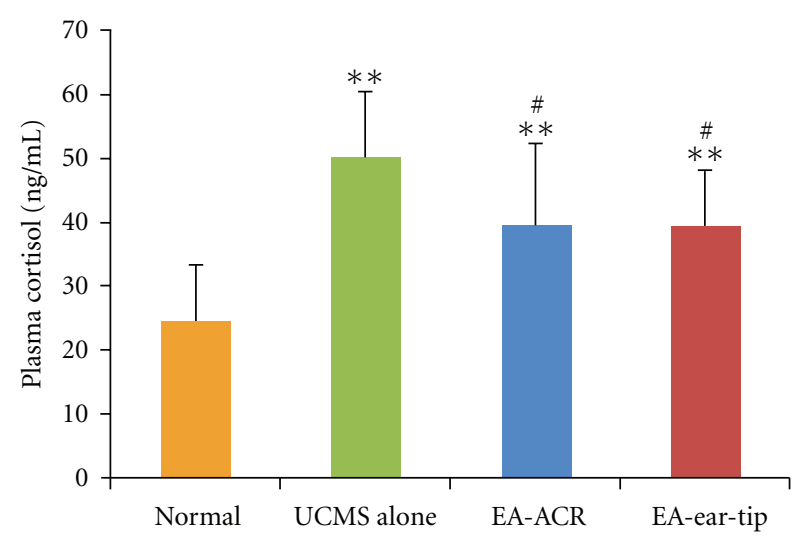

FIgURE 5: The effect of EA-ACR treatment on plasma cortisol in normal and UCMS rats. Comparison of plasma cortisol level between UCMS and normal groups, ${ }^{* *} P<0.01$. Comparison between the EA-treated UCMS group and the UCMS alone group, ${ }^{\#} P<0.05$.

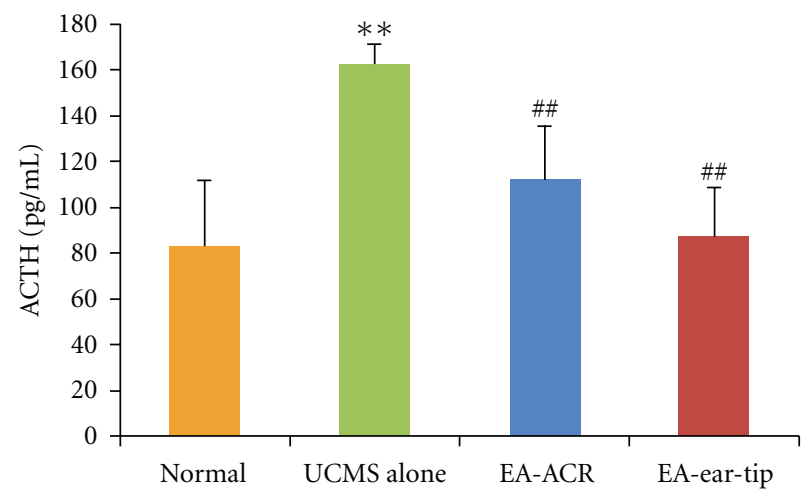

FIGURE 6: The effect of EA-ACR treatment on ACTH in normal and UCMS rats. Comparison of the ACTH level between normal and UCMS groups, ${ }^{* *} P<0.01$. Comparison between the EA-treated UCMS group and the UCMS alone group, ${ }^{\# \#} P<0.01$.

The result of the present study-EA-ACR significantly antagonized UCMS-induced depressive status of rats-is consistent with the findings of the mentioned research studies. As demonstrated through changes in plasma cortisol and ACTH levels, the antidepressant effect of EA-ACR may be mediated via normalization of the HPA axis hyperactivity. Otherwise, EA-ear-tip also was found to be the apparent down-regulation effect on the plasma cortisol and ACTH. It is found that a few vagus nerve fibers are around the ear tip [30], and HPA may be modulated by other nervous pathways beside the vagus nerve, for example, greater auricular nerve and lesser occipital nerve are densely innervated in the area of ear tip, and the EA signals can be transmitted by them to the cervical spinal cord and brain then modulate the HPA. Further investigation has been warranted for this hypothesis.

\section{Limitation}

This pilot EA-ACR study on depression has a small sample size. Meanwhile, EA-ACR does not only stimulate the 
vagus nerve, but also affects other sensory nerves, such as nervous auricularis magnus, lesser occipital nerve, facial nerve, and glossopharyngeal nerve fibers. Although EA-ACR elicited similar effects to VNS, the interaction among the nerves in the area should be explored in the future. Further investigation on EA-ACR for the disturbed monoaminergic neurotransmission of depression has been warranted.

\section{Conclusions}

EA-ACR can elicit similar cardioinhibitory effects to vagus nerve stimulation (VNS), and EA-ACR significantly antagonized UCMS-induced depressive status of rats. The antidepressant effect of EA-ACR is possibly mediated via normalization of the HPA axis hyperactivity.

\section{Author's Contribution}

Ru-Peng Liu and Ji-Liang Fang contributed equally to this paper.

\section{Acknowledgments}

This scientific work was supported by the National Natural Science Foundation of China Research Grants (no. 30973798), the Twelfth Five-Year Plan of the National Science and Technology Support Program of China (2012BAF14B10), and the Beijing Natural Science Foundation (no. 711117).

\section{References}

[1] H. A. Sackeim, A. J. Rush, M. S. George et al., "Vagus nerve stimulation (VNSÜ) for treatment-resistant depression: efficacy, side effects, and predictors of outcome," Neuropsychopharmacology, vol. 25, no. 5, pp. 713-728, 2001.

[2] A. J. Rush, L. B. Marangell, H. A. Sackeim et al., "Vagus nerve stimulation for treatment-resistant depression: a randomized, controlled acute phase trial," Biological Psychiatry, vol. 58, no. 5, pp. 347-354, 2005.

[3] A. J. Rush, H. A. Sackeim, L. B. Marangell et al., "Effects of 12 months of vagus nerve stimulation in treatment-resistant depression: a naturalistic study," Biological Psychiatry, vol. 58, no. 5, pp. 355-363, 2005.

[4] A. A. Nierenberg, J. E. Alpert, E. E. Gardner-Schuster, S. Seay, and D. Mischoulon, "Vagus nerve stimulation: 2-year outcomes for bipolar versus unipolar treatment-resistant depression," Biological Psychiatry, vol. 64, no. 6, pp. 455-460, 2008.

[5] M. S. George, H. A. Sackeim, A. J. Rush et al., "Vagus nerve stimulation: a new tool for brain research and therapy," Biological Psychiatry, vol. 47, no. 4, pp. 287-295, 2000.

[6] M. J. Millan, "The role of monoamines in the actions of established and "novel" antidepressant agents: a critical review," European Journal of Pharmacology, vol. 500, no. 1-3, pp. 371384, 2004.

[7] W. Sperling, U. Reulbach, S. Bleich, F. Padberg, J. Kornhuber, and M. Mueck-Weymann, "Cardiac effects of vagus nerve stimulation in patients with major depression," Pharmacopsychiatry, vol. 43, no. 1, pp. 7-11, 2010.
[8] S. Spuck, V. Tronnier, I. Orosz et al., "Operative and technical complications of vagus nerve stimulator implantation," Neurosurgery, vol. 67, no. 2, pp. 489-494, 2010.

[9] X. Y. Gao, Y. H. Li, P. J. Rong, and B. Zhu, "Effect of acupuncture of auricular concha area on blood pressure in primary hypertension and normal rats and analysis on its mechanism," Acupuncture Research, vol. 31, pp. 90-95, 2006.

[10] X. Y. Gao, Y. H. Li, K. Liu et al., "Acupuncture-like stimulation at auricular point Heart evokes cardiovascular inhibition via activating the cardiac-related neurons in the nucleus tractus solitarius," Brain Research, vol. 1397, pp. 19-27, 2011.

[11] Z. G. Mei, B. Zhu, Y. H. Li, P. J. Rong, H. Ben, and L. Li, "Responses of glucose-sensitive neurons and insulin-sensitive neurons in nucleus tractus solitarius to electroacupuncture at auricular concha in rats," Chinese Acupuncture \& Moxibustion, vol. 27, no. 12, pp. 917-922, 2007.

[12] F. Huang, P. J. Rong, H. C. Wang, H. Meng, and B. Zhu, "Clinical observation on the intervention of auricular vagus nerve stimulation treating 35 cases of impaired glucose tolerance patients," China Journal of Traditional Chinese Medicine and Pharmacy, vol. 25, no. 12, pp. 2185-2186, 2010.

[13] W. He, C. L. Zhao, Y. H. Li et al., "Effect of electroacupuncture at auricular concha on behaviors and electroencephalogram in epileptic rats," Chinese Journal of Pathophysiology, vol. 27, no. 10, pp. 1913-1916, 2011.

[14] W. He, Y. H. Li, P. J. Rong, L. Li, H. Ben, and B. Zhu, "Effect of electroacupuncture of different regions of the auricle on epileptic seizures in epilepsy rats," Acupuncture Research, vol. 38, no. 6, pp. 417-422, 2011.

[15] Tang. Wang Bing. Inner Canon of Huang Di. Chinese Ancient Books Publishing House Yin the Qing Dynasty Jingkou Wencheng Tang inscription of Song, Beijing, China, 2003.

[16] J. J. B. Allen, R. N. Schnyer, and S. K. Hitt, "The efficacy of acupuncture in the treatment of major depression in women," Psychological Science, vol. 9, no. 5, pp. 397-401, 1998.

[17] H. C. Luo, H. Ureil, Y. C. Shen et al., "Comparative study of electroacupuncture and fiuoxetine for treatment of depression," Chinese Journal of Psychiatry, vol. 36, pp. 215-219, 2003.

[18] W. J. Zhang, X. B. Yang, and B. L. Zhong, "Combination of acupuncture and fluoxetine for depression: a randomized, double-blind, sham-controlled trial," Journal of Alternative and Complementary Medicine, vol. 15, no. 8, pp. 837-844, 2009.

[19] H. Wang, H. Qi, B. S. Wang et al., "Is acupuncture beneficial in depression: a meta-analysis of 8 randomized controlled trials?" Journal of Affective Disorders, vol. 111, no. 2-3, pp. 125-134, 2008.

[20] Y. L. Sun, S. B. Chen, Y. Gao, and J. Xiong, "Acupuncture versus western medicine for depression in China: a systematic review," Chinese Journal of Evidence-Based Medicine, vol. 8, no. 5, pp. 340-345, 2008.

[21] J. N. Ren, "Auricular acupuncture to treat depression, 50 cases," Henan Traditional Chinese Medicine, vol. 25, no. 2, pp. 75-78, 2005.

[22] Y. M. Liu and H. P. Su, "Observation of clinical efficacy on acupuncture with auricular acupressure to treatment of depression," Liaoning Journal of Traditional Chinese Medicine, vol. 35, no. 1, pp. 122-125, 2008.

[23] J. M. Li, "Observation of clinical curative effect on the treatment of 35 cases of depression with acupuncture and auricular therapy," Journal of Yunnan University of Traditional Chinese Medicine, vol. 33, no. 1, pp. 5-6, 2010. 
[24] R. J. Katz, K. A. Roth, and B. J. Carroll, "Acute and chronic stress effects on open field activity in the rat: implications for a model of depression," Neuroscience \& Biobehavioral Reviews, vol. 5, pp. 247-251, 1981.

[25] D. M. Duan, Y. Tu, and L. P. Chen, "Effects of electroacupuncture at different acupoint groups on behavior activity and pCREB expression in hippocampus in the rat of depression," Chinese Acupuncture \& Moxibustion, vol. 28, no. 5, pp. 369-373, 2008.

[26] P. Willner, "Validity, reliability and utility of the chronic mild stress model of depression: a 10-year review and evaluation," Psychopharmacology, vol. 134, no. 4, pp. 319-329, 1997.

[27] D. A. Groves and V. J. Brown, "Vagal nerve stimulation: a review of its applications and potential mechanisms that mediate its clinical effects," Neuroscience and Biobehavioral Reviews, vol. 29, no. 3, pp. 493-500, 2005.

[28] T. Kawada, S. Shimizu, M. Li et al., "Contrasting effects of moderate vagal stimulation on heart rate and carotid sinus baroreflex-mediated sympathetic arterial pressure regulation in rats," Life Sciences, vol. 89, no. 13, pp. 498-503, 2011.

[29] H. J. Sun, H. B. Ai, and X. Y. Cui, "Effects of electrical stimulation to the dorsal motor vagal nudens ucleus on HEECG signals," Journal of Shanxi University, vol. 27, pp. 289-291, 2004.

[30] S. Standring, Gray's Anatomy, Churchill Livingstone, New York, NY, USA, 40th edition, 2008.

[31] Y. S. Mineur, C. Belzung, and W. E. Crusio, "Effects of unpredictable chronic mild stress on anxiety and depression-like behavior in mice," Behavioural Brain Research, vol. 175, no. 1, pp. 43-50, 2006.

[32] M. P. Boyle, J. A. Brewer, M. Funatsu et al., "Acquired deficit of f orebrain glucocort icoid recept or produces depression-like changes in adrenal axis regulation and behavior," Proceedings of the National Academy of Sciences of the United States of America, vol. 102, no. 2, pp. 473-478, 2005.

[33] H. Xu, Z. R. Sun, L. P. Li et al., "Effect s of acupuncture on the hypothalamus- pituitary-adrenal axis in the patients of depression," Chinese Acupuncture and Moxibustion, vol. 24, no. 2, pp. 78-80, 2004.

[34] C. B. Nemeroff, H. S. Mayberg, S. E. Krahl et al., "VNS therapy in treatment-resistant depression: clinical evidence and putative neurobiological mechanisms," Neuropsychopharmacology, vol. 31, no. 7, pp. 1345-1355, 2006.

[35] J. P. Herman, H. Figueiredo, N. K. Mueller et al., "Central mechanisms of stress integration: hierarchical circuitry controlling hypothalamo-pituitary-adrenocortical responsiveness," Frontiers in Neuroendocrinology, vol. 24, no. 3, pp. 151-180, 2003.

[36] N. Barden, "Implication of the hypothalamic-pituitary-adrenal axis in the physiopathology of depression," Journal of Psychiatry and Neuroscience, vol. 29, no. 3, pp. 185-193, 2004.

[37] S. M. O’Toole, L. K. Sekula, and R. T. Rubin, "Pituitary-adrenal cortical axismeasures as predictors of sustainedremission in major depression," Biological Psychiatry, vol. 42, pp. 85-89, 1997.

[38] V. O’Keane, T. G. Dinan, L. Scott, and C. Corcoran, "Changes in hypothalamic-pituitary-adrenal axis measures after vagus nerve stimulation therapy in chronic depression," Biological Psychiatry, vol. 58, no. 12, pp. 963-968, 2005. 


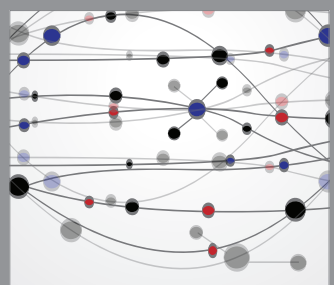

The Scientific World Journal
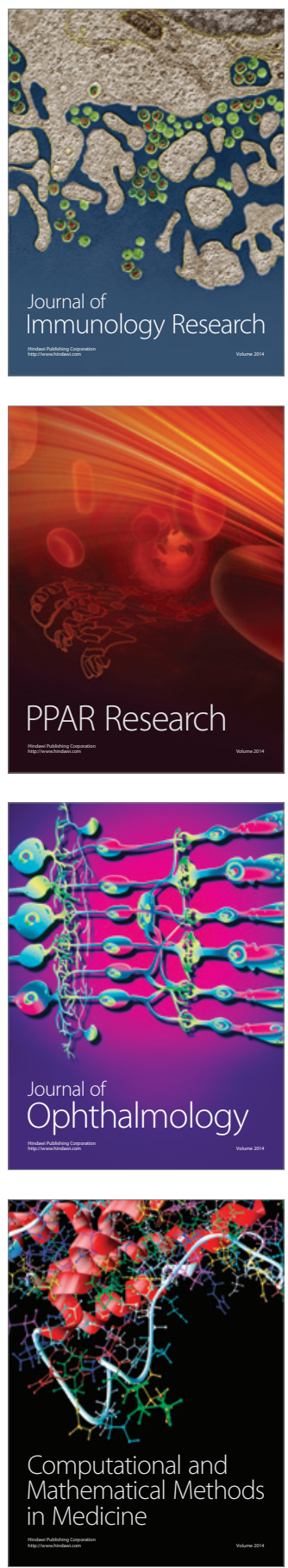

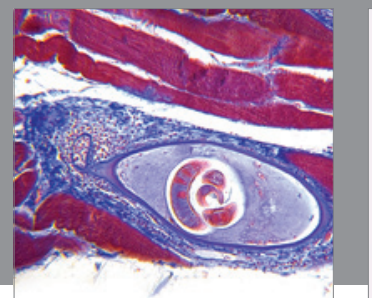

Gastroenterology

Research and Practice
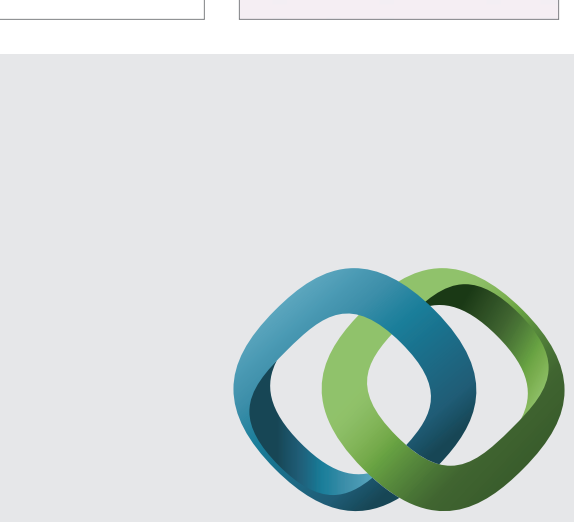

\section{Hindawi}

Submit your manuscripts at

http://www.hindawi.com
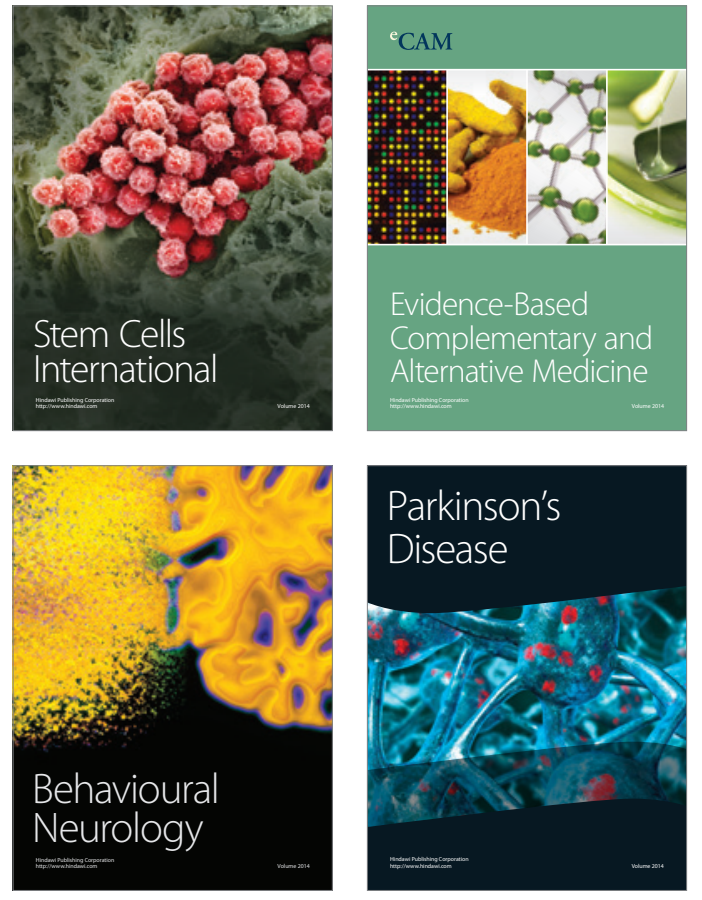
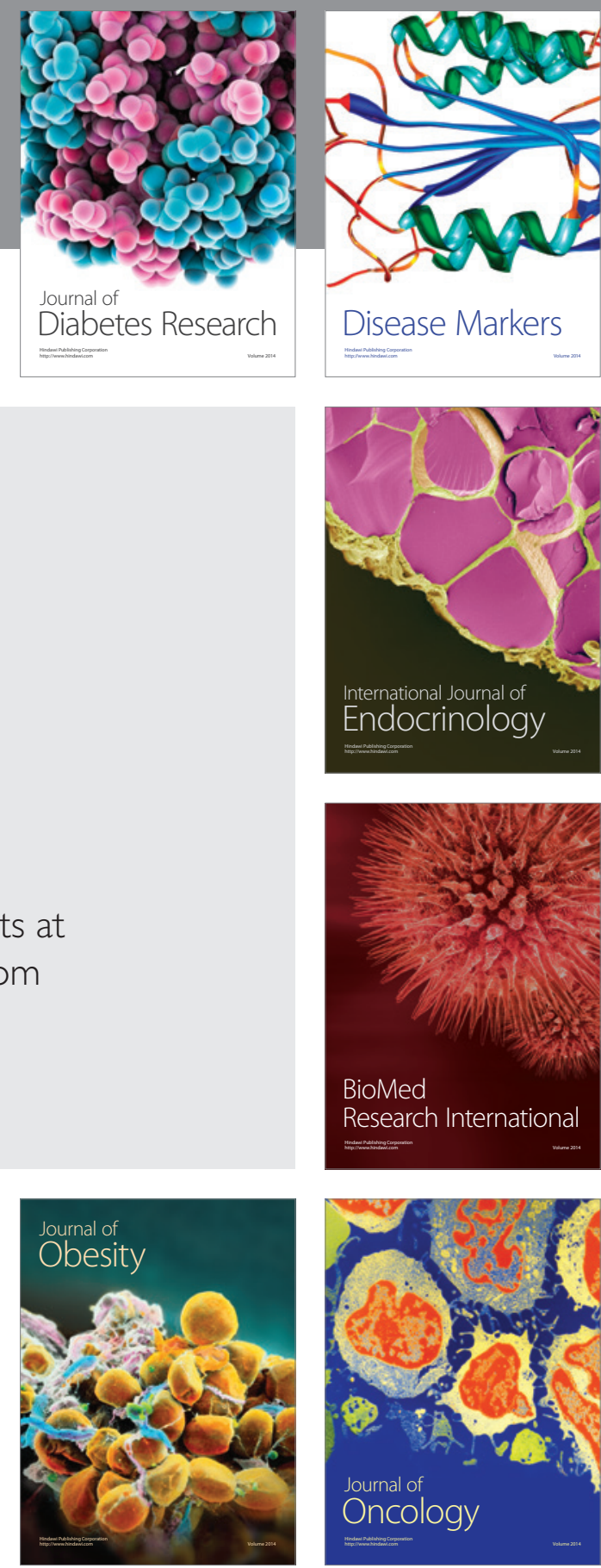

Disease Markers
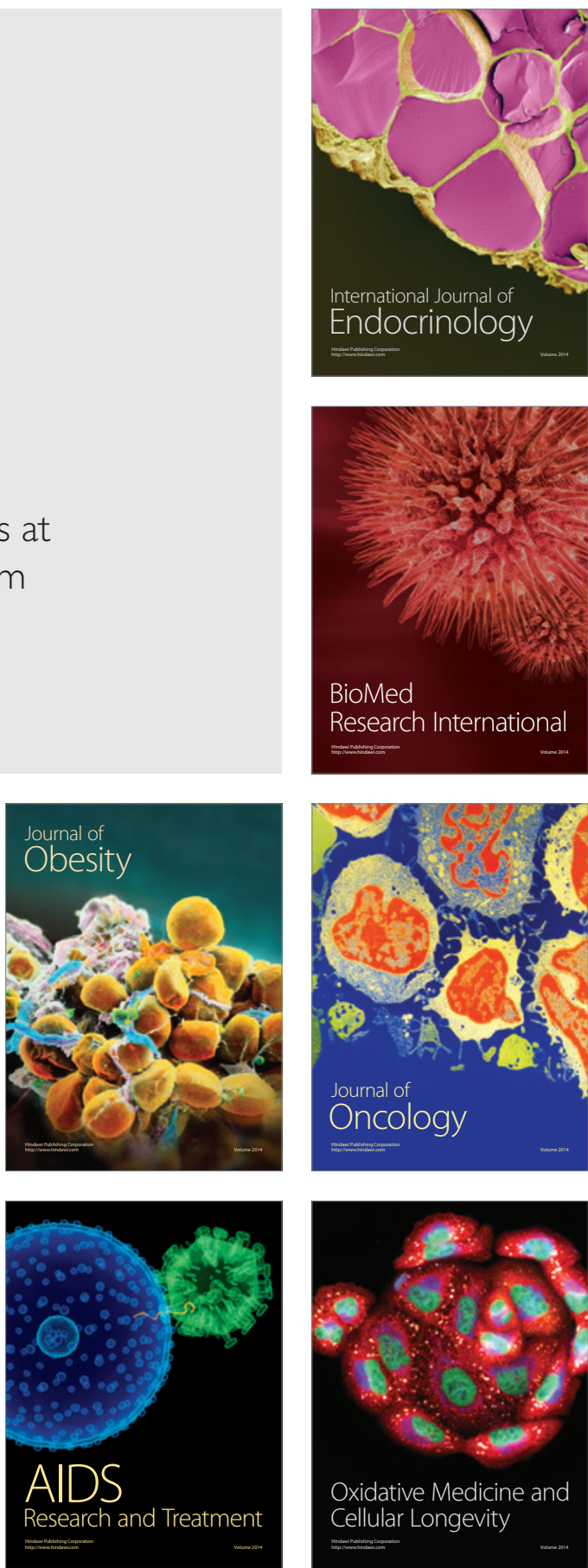